\title{
DEFICIÊNCIA PROGRESSIVA DOS HORMÔNIOS ADENO-HIPOFISÁRIOS APÓS RADIOTERAPIA EM ADULTOS*
}

\author{
Rafaela A. Loureiro ${ }^{1}$, Mário Vaisman ${ }^{2}$
}

Resumo A radioterapia é um dos fatores desencadeantes do hipopituitarismo, mesmo quando não direcionada diretamente para o eixo hipotálamo-hipofisário, podendo resultar em redução de hormônios adeno-hipofisários, principalmente por lesão hipotalâmica. A perda da função da hipófise anterior é progressiva e geralmente na seguinte ordem: hormônio do crescimento, gonadotrofinas, adrenocorticotrofina e o hormônio estimulante da tireóide. Vários testes estão disponíveis para a confirmação das deficiências, sendo discutidos, neste artigo, os melhores testes para pacientes submetidos à irradiação. Enfatizamos que o desenvolvimento do hipopituitarismo após a radioterapia é dose e tempo dependente de irradiação, com algumas diferenças entre os eixos hipofisários. Portanto, a conscientização da necessidade de terapia em conjunto de endocrinologistas e oncologistas otimizará o tratamento e a qualidade de vida do paciente.

Unitermos: Radioterapia; Hipopituitarismo; Adeno-hipófise; Diagnóstico; Qualidade de vida.

Abstract Progressive pituitary hormone deficiency following radiation therapy in adults.

Hypopituitarism can be caused by radiation therapy, even when it is not directly applied on the hypothalamic-pituitary axis, and can lead to anterior pituitary deficiency mainly due to hypothalamic damage. The progressive loss of the anterior pituitary hormones usually occurs in the following order: growth hormone, gonadotropin hormones, adrenocorticotropic hormone and thyroid-stimulating hormone. Although there are several different tests available to confirm anterior pituitary deficiency, this paper will focus on the gold standard tests for patients submitted to radiation therapy. We emphasize that the decline of anterior pituitary function is time- and dose-dependent with some variability among the different axes. Therefore, awareness of the need of a joint management by endocrinologists and oncologists is essential to improve treatment and quality of life of the patients.

Key words: Radiotherapy; Hypopituitarism; Anterior pituitary; Diagnosis; Quality of life.

\section{INTRODUÇÃO}

A deficiência de hormônios adeno-hipofisários pode resultar de causas hereditárias ou adquiridas. Dentre as adquiridas, devemos considerar as etiologias traumáticas (irradiação, cirurgia), neoplásicas (tumores hipotálamo-hipofisários, cistos), vasculares (síndrome de Sheehan, apoplexia), infecciosas (tuberculose, histoplasmose), infiltrativas (hipofisite), funcionais (exercício excessivo, nutricional) e através de drogas (estrogênio, esteróides anabolizantes, excesso de glicocorticóides e hor-

* Trabalho realizado no Serviço de Endocrinologia do Hospital Universitário Clementino Fraga Filho (HUCFF) da Universidade Federal do Rio de Janeiro (UFRJ), Rio de Janeiro, RJ.

1. Pesquisadora Assistente do Serviço de Endocrinologia do HUCFF-UFRJ, Fellowship em Endocrinologia no Saint Bartholo mew's Hospital, Londres, Reino Unido.

2. Professor Adjunto Doutor da Faculdade de Medicina da UFRJ, Chefe do Serviço de Endocrinologia do HUCFF-UFRJ.

Endereço para correspondência: Dra. Rafaela A. Loureiro. Rua Teixeira Mendes, 153, ap. 404, Laranjeiras. Rio de Janeiro, RJ, 22245-090. E-mail: rafaela_loureiro@hotmail.com

Recebido para publicação em 4/8/2003. Aceito, após revisão, em 10/10/2003. mônios tireoidianos, dopamina, análogos da somatostatina) $)^{(\mathbf{1})}$.

O objetivo deste artigo é enfocar a radioterapia como fator desencadeante de hipopituitarismo. A radioterapia pode provocar deficiências multi-hormonais, tanto se direcionada diretamente para o eixo hipotálamo-hipofisário no tratamento de tumores da região quanto para outros locais, como no tratamento de câncer nasofaríngeo, tumores cerebrais, irradiação profilática para leucemia linfoblástica aguda ou irradiação corporal total para uma variedade de tumores e outras doenças. Nesse caso, pode-se calcular a dose efetiva biológica de radioterapia sobre o eixo hipotálamo-hipofisário ${ }^{(2)}$.

\section{AVALIAÇÃO FUNCIONAL DA HIPÓFISE}

A função da hipófise anterior diminui com o passar dos anos após a radioterapia, predominantemente como resultado de lesão hipotalâmica. A perda progressiva dos hormônios hipofisários anteriores em pacientes submetidos à radioterapia se dá usualmente na seguinte ordem: hormônio do crescimento $(\mathrm{GH})$, gonadotrofinas (LH/ FSH), adrenocorticotrofina (ACTH) e hormônio estimulante da tireóide (TSH).

$\mathrm{O}$ eixo hormônio estimulante de $\mathrm{GH}$ (GHRH)/GH é o mais vulnerável à radioterapia. A chance de deficiência de GH aumenta com o tempo pós-radioterapia, sendo dependente do status antes do procedimento terapêutico, ou seja, quanto maior o pico de $\mathrm{GH}$ após estímulo antes da radioterapia, maior será o tempo que o paciente levará para se tornar deficiente. $\mathrm{O}$ pico de resposta do GH ao teste de tolerância à insulina (ITT) declina principalmente durante os primeiros cinco anos após a radioterapia, chegando então a um platô. Certas variações devem ser levadas em conta, como idade, sexo e se o paciente foi submetido à cirurgia antes da radioterapia. Na literatura não há um consenso sobre o efeito da idade na resposta de $\mathrm{GH}$, já que alguns resultados demonstram que não há 
mudança, e outros, que há redução. Em relação ao sexo, as mulheres mostram maiores picos de $\mathrm{GH}$ do que os homens, e a cirurgia diminui a resposta na maioria dos pacientes $^{(3)}$.

Como já mencionado, a lesão hipotalâmica ocorre primeiramente, causando deficiência do GHRH e da somatostatina. A lesão somatotrófica ocorre tardiamente, refletindo atrofia somatotrófica secundária. Por isso, é preferível para o diagnóstico precoce de deficiência de GH o ITT, em vez do teste de estímulo com arginina (AST) + GHRH. A disfunção somatotrófica pode ser atribuída à atrofia secundária à diminuição de GHRH ou a efeito tardio direto da radioterapia sobre as células somatotróficas. Isto pode ser verificado por um aumento de prolactina após irradiação, seguido de diminuição dela com o passar dos anos, refletindo primeiramente deficiência do fator inibidor de prolactina (PIF), levando à hiperprolactinemia, e posteriormente, disfunção das células lactotróficas, levando a um declínio da prolactina ${ }^{(2)}$.

Devemos lembrar, no entanto, que a secreção de GH se mantém pulsátil, a variação diurna de GH está reduzida, mas não ausente, e que a maior alteração é a redução da amplitude do pulso de $\mathrm{GH}$, mesmo após irradiação, doenças hipofisárias e cirurgia do eixo hipotálamo-hipofisário ${ }^{(4)}$.

O teste considerado padrão ouro para diagnosticar a deficiência de GH em pacientes submetidos à radioterapia é o ITT. Devemos ressaltar que a hipoglicemia deverá ser constatada clinicamente e bioquimicamente, caso contrário o teste não terá validade. Nos casos de contra-indicação ao teste (doença coronariana, epilepsia, hipoadrenalismo), o preferido é o teste do glucagon $^{(3)}$. A melhor sensibilidade desses testes provavelmente deve-se ao fato de agirem via receptores $\alpha$-adrenérgicos. $\mathrm{O}$ mecanismo de ação do ITT ainda não é totalmente entendido. Além da ação via receptores $\alpha$-adrenérgicos, outros mecanismos podem estar envolvidos, como a supressão de somatostatina e a secreção de GHRH. A porção basomedial do hipotálamo provavelmente contém neurônios responsáveis em provocar respostas neuroendócrinas à hipoglicemia ${ }^{(5)}$. Ainda é preferido, devido ao fato já explicado de a irradiação causar lesão primariamente hipo- talâmica, ficando assim vários testes com menor poder diagnóstico logo após a terapia, como GHRH, GHRH + GHRP-6 (hexarelina) $)^{(2)}$.

O teste da arginina induz à secreção de $\mathrm{GH}$, inibindo a secreção de somatostatina. Também age através de receptores $\alpha$-adrenérgicos. No entanto, já foi demonstrado que a resposta de $\mathrm{GH}$ a esse teste parece ser mais resistente aos efeitos da irradiação que o ITT. Além disso, já foi esclarecido que indivíduos normais apresentam uma resposta mais exuberante com o ITT $^{(5)}$.

Existem vários outros testes provocativos de GH, mas que iremos apenas citar pelo fato de não apresentarem melhor resposta que o ITT em pacientes submetidos à irradiação $(\text { Tabela } 1)^{(\mathbf{6})}$.

Tabela 1 Testes utilizados na comprovação de deficiências adeno-hipofisárias.

\begin{tabular}{|l|l|}
\hline \multicolumn{1}{|c|}{ Eixo } & \multicolumn{1}{|c|}{ Possíveis testes } \\
\hline GH & $\begin{array}{l}\text { ITT, glucagon, arginina, arginina + } \\
\text { GHRH, arginina + L-dopa, ornitina, } \\
\text { clonidina (crianças), L-dopa, GHRH, } \\
\\
\text { GHRP-6, GHRH + GHRP-6 }\end{array}$ \\
FSH/LH & GnRH \\
ACTH & ITT, CRH, ACTH, metirapona \\
TSH & TRH \\
\hline
\end{tabular}

GH, hormônio de crescimento; ITT, teste de tolerância à insulina; GHRH, hormônio estimulante de GH; L-dopa, levodopa; GHRP-6, hexarelina; FSH/LH, gonadotrofinas; GnRH, hormônio estimulador de gonadotrofinas; ACTH, adrenocorticotrofina; $\mathrm{CRH}$, hormônio liberador da corticotrofina; TSH, hormônio estimulante da tireóide; TRH, hormônio estimulador da tireotrofina.

Em relação a outros possíveis marcadores, como IGF-I (fator insulina símile tipo I, IGFBP-3 (proteína carreadora tipo 3) e GHBP (proteína carreadora de GH), sabese que não podem ser utilizados como parâmetro de definição de deficiência isoladamente. Níveis de IGF-I encontram-se diminuídos, mas geralmente os valores de IGFBP-3 estão normais. Em um estudo em que pacientes foram submetidos a radioterapia e quimioterapia, houve declínio paralelo entre eles, principalmente durante os seis primeiros meses após a radio/quimioterapia, em contraste com valores normais de $\mathrm{GH}$ a teste provocativo, demonstrando possível período transitório de insensibilidade de GH. Porém, faltam estudos mais conclusivos sobre esse período de insensibilidade em pacientes submetidos apenas à irradiação ${ }^{(7)}$.
Por outro lado, em pacientes que apresentam outras deficiências hormonais da adeno-hipófise e ainda apresentam baixos valores de IGF-I, fica praticamente evidente a deficiência de GH. Muitas autoridades de saúde do mundo exigem como controle de liberação para terapia com $\mathrm{GH}$, que pelo menos dois testes sejam realizados para comprovar a deficiência, por causa do seu alto custo. No Brasil, o governo apenas libera a terapia com GH para pacientes em fase de crescimento ${ }^{(\mathbf{1})}$.

A disfunção dos demais eixos pode ser demonstrada também, na maioria das vezes, em um ano após a irradiação. No eixo hormônio estimulador de gonadotrofinas (GnRH)/FSH-LH, verificamos predominantemente deficiência de GnRH. Em resposta ao análogo do GnRH, foi demonstrado que há mudanças discordantes entre FSH e LH. Geralmente, o FSH mostra-se elevado e o LH diminuído, em função da redução da freqüência de pulso do $\mathrm{GnRH}^{(\mathbf{8})}$. Alguns pacientes podem mostrar FSH e LH normais ou pouco abaixo dos níveis de referência. As concentrações de estradiol ou testosterona apresentam-se reduzidas. Obviamente, os resultados podem variar bastante quando os pacientes recebem irradiação na fase pré-puberal, influenciando inclusive no grau de desenvolvimento puberal. A capacidade da hipófise de responder a análogos do GnRH demonstra hipogonadismo hipotalâmico ${ }^{(9)}$.

O teste do GnRH não apenas diagnostica a deficiência de gonadotrofinas, mas também avalia o nível de reserva de FSH/ LH. Deve ser lembrado que o teste também deve ser realizado preferencialmente na fase folicular do ciclo menstrual ${ }^{(\mathbf{9})}$.

O eixo hipotálamo-hipófise-adrenal é o penúltimo a demonstrar disfunção. Dessa forma, a deficiência de cortisol é detectada mais tardiamente através do ITT, não associando a etiologia ao hipotálamo ou à hipófise. Mas, como já demonstrado através dos demais eixos, provavelmente a anormalidade primária é hipotalâmica ${ }^{(3)}$.

Podem ser feitos, ainda, o teste do hormônio liberador da corticotrofina (CRH), o teste da corticotrofina e o teste da metirapona. $\mathrm{O}$ teste do $\mathrm{CRH}$ causará aumento exagerado de ACTH em comprometimento hipotalâmico e sem resposta em acometimento hipofisário. Já o teste da cortico- 
trofina só verificará a presença de problema hipofisário, e ainda, alguns pacientes deficientes em ACTH mostram resposta normal de cortisol a este teste, mas respostas subnormais ao ITT ou ao teste da metirapona. Este último mostra baixos níveis de 11-deoxicortisol em pacientes deficientes de $\mathrm{ACTH}^{(\mathbf{9})}$.

Já a deficiência de hormônio estimulador da tireotrofina (TRH) é bem documentada através da resposta tardia de TSH ao TRH, de concentrações baixas de T3, T4 livre e total, sugerindo defeito da liberação de TRH. Foram levantadas as hipóteses de que esses achados resultariam de lesão direta às células responsáveis pela secreção hormonal, lesão ao estroma ou microvasculatura, ou ainda lesão dos canais vasculares que transportam os hormônios hipotalâmicos para a hipófise ${ }^{(9)}$.

O teste do TRH poderá ser feito isoladamente ou junto com o teste do GnRH (megateste). O paciente deve ser alertado de possíveis efeitos colaterais: náusea, vermelhidão em face e desejo de urinar. $\mathrm{O}$ envolvimento hipofisário poderá ser verificado através de baixos valores de T4 livre e níveis normais ou baixos de $\mathrm{TSH}^{(\mathbf{1 0})}$.

Os testes para confirmação de deficiências adeno-hipofisárias devem ser feitos anualmente após irradiação de cabeça, pescoço e sistema nervoso central do paciente, por pelo menos dez anos após a terapia ou até a detecção e tratamento de certa deficiência $^{(11)}$.

\section{TRATAMENTO}

A clínica de pan-hipopituitarismo pósradioterapia é bem reconhecida, já que não difere como resultado de outras etiologias (Tabela 2) ${ }^{(\mathbf{1})}$.
O tratamento deve ser iniciado tão logo sejam detectadas as deficiências. O tratamento de deficiências adeno-hipofisárias por irradiação não difere das demais etiologias. No entanto, algumas observações serão feitas ${ }^{(11)}$.

Sempre antes de iniciar a reposição com tiroxina deve-se iniciar a reposição com glicocorticóide (a tiroxina pode exacerbar a deficiência de glicocorticóide). Os esteróides sexuais seriam seguidos da tiroxina, e por último seria o tratamento com $\mathrm{GH}$.

A reposição de $\mathrm{GH}$ ativa a desiodação de tiroxina e estimula a conversão de tiroxina para triiodotironina. Porém, essas mudanças são transitórias e não requerem ajuste da reposição com tiroxina. Já em relação ao eixo adrenocorticotrófico, a reposição com $\mathrm{GH}$ poderá diminuir a hidrocortisona disponível, provavelmente por uma modulação da atividade da enzima $11 \beta$ hidroxiesteróide desidrogenase mediada por GH ou IGF-I ${ }^{(\mathbf{1})}$. O tratamento com GH não demonstrou recorrência de tumor prévio na região hipotálamo-hipofisária em pacientes submetidos à radioterapia do eixo em um período de até quatro anos pós-radioterapia. A reposição foi iniciada tão logo detectada deficiência através do ITT $^{(\mathbf{1 2})}$. Outro ponto importante é a queda de concentrações de IGF-I após o início da reposição com estrogênio oral, devendo ser iniciado, em pacientes severamente deficientes em $\mathrm{GH}$, o adesivo transdérmico ou percutâneo, vias nas quais o estrogênio não será metabolizado pelo fígado ${ }^{(\mathbf{1})}$.

\section{RADIOTERAPIA E HIPOPITUITARISMO}

Além do hipopituitarismo, os efeitos colaterais da radioterapia incluem distúr-

Tabela 2 Resumo do quadro clínico do pan-hipopituitarismo.

\begin{tabular}{|l|l|}
\hline Deficiência & \multicolumn{1}{c|}{ Quadro clínico } \\
\hline GH & $\begin{array}{l}\text { Baixo nível de energia, diminuição de densidade óssea, aumento da gordura corporal, } \\
\text { aterogênese acelerada com aumento do colesterol LDL e Apo B, diminuição do coles- } \\
\text { terol HDL, diminuição de sensibilidade à insulina e aumento da prevalência de intole- } \\
\text { rância à glicose } \\
\text { FSH/LH }\end{array}$ \\
$\begin{array}{l}\text { Infertilidade, diminuição de libido e potência em homens, ondas de calor e resseca- } \\
\text { mento vaginal em mulheres }\end{array}$ \\
ACTH & $\begin{array}{l}\text { Náuseas, vômitos, desconforto epigástrico, cefaléia, hipotensão postural, hipoglicemia } \\
\text { TSH }\end{array}$ \\
& Distúrbios menstruais (amenorréia ou menorragia), ressecamento da pele e cabelos, \\
\hline
\end{tabular}

GH, hormônio de crescimento; FSH/LH, gonadotrofinas; ACTH, adrenocorticotrofina; TSH, hormônio estimulante da tireóide. bios visuais (cegueira) por lesão do nervo óptico, carcinogênese cerebral secundária e isquemia ou necrose cerebral. A neuropatia óptica por irradiação é um acontecimento raro após radioterapia convencional, sendo mais freqüente em pacientes submetidos a cirurgia, seguida de radioterapia convencional e/ou radiocirurgia ${ }^{(\mathbf{1 3})}$. Já foi documentado, após irradiação do couro cabeludo em um mesmo paciente, o aparecimento de prolactinoma, adenoma de paratireóide, lesão benigna de tireóide e carcinoma basocelular da pele. A análise molecular não revelou nenhuma anormalidade para neoplasia endócrina múltipla tipo 1 (MEN-1) ${ }^{(\mathbf{1 4})}$. Outro achado curioso em pacientes submetidos à radioterapia foi a constatação de hiperleptinemia, que possivelmente ocorre como conseqüência de lesão hipotalâmica induzida por irradiação ou de deficiência de $\mathrm{GH}^{(\mathbf{1 5})}$. Após radioterapia do eixo hipotálamo-hipofisário, foram constatadas mudanças histológicas da hipófise. A fibrose foi associada a um aumento no número de células folículo-estelares, disfunção mitocondrial e metaplasia escamosa. Esses achados são predominantes em células hipofisárias irradiadas, podendo participar na hipofunção hipofisária tardia. Nas células de adenomas irradiados, a fibrose difusa está ausente e as mudanças histológicas são maiores após radiocirurgia ("gamma knife") do que em relação à radioterapia convencional ${ }^{(16)}$.

O desenvolvimento do hipopituitarismo após a radioterapia é dose/tempo dependente de irradiação. Já foi demonstrado que a deficiência de $\mathrm{GH}$ não é frequiente com doses abaixo de $20 \mathrm{~Gy}$, por outro lado, sendo quase uma constante quando doses maiores que 45 Gy são utilizadas. Porém, já foi mostrada deficiência de GH com doses de 18 Gy. Já em relação ao tempo de deficiência de GH pós-radioterapia, entende-se que o maior declínio de GH ocorre nos primeiros cinco anos, levando-se em conta também a resposta de $\mathrm{GH}$ antes e logo após à radioterapia. Em relação aos demais hormônios hipofisários, dose de 36 Gy ou menos resulta quase sempre em hipotireoidismo, não havendo uma dosagem específica para deficiência de LH/FSH e ACTH. Hiperprolactinemia é sempre vista com doses maiores que $40 \mathrm{~Gy}$, quando o eixo hipotálamo-hipófise é incluído no 
campo de tratamento. Qualquer paciente que receba dose total de irradiação de 20 Gy ou mais direcionada ao eixo terá risco de manifestar hipopituitarismo ${ }^{(3,17)}$.

Foi demonstrado, em um estudo que utilizou radioterapia com raios "protonphoton" (PPRT) direcionados para a base do crânio, em que a média da dose prescrita era 68,4 CGE ("cobalt gray equivalent”), que mesmo uma dose alvo mínima era preditiva de deficiências adeno-hipofisárias. Pacientes que receberam dose de 50 CGE em relação à hipófise experimentaram maior incidência e gravidade das disfunções adeno-hipofisárias. Dose de 70 CGE ou mais em relação à hipófise e de 50 CGE ou mais em ralação ao hipotálamo eram preditivas de maior acometimento de distúrbio endócrino ${ }^{(\mathbf{1 8})}$.

Outro estudo em crianças propôs uma equação com o objetivo de prever as mudanças no pico de GH num período de 0 12 meses após radioterapia convencional, de acordo com as doses 0-20 Gy, 20-40 Gy e 40-60 Gy. Foi assumido que o efeito integral de irradiação era uma soma linear dos produtos de cada volume de dose e assim era avaliado o impacto de cada intervalo de dose. Como esperado, o efeito do intervalo de dose de $0-20$ foi menor que os demais, no que se refere aos picos de GH. Segue a equação utilizada:

$$
\begin{gathered}
G H(t)=\operatorname{Exp}(\ln (b G H)-(0,00058 \mathrm{~V} \\
(0-20 G y)+0,00106 V(20-40 G y)+ \\
0,00156 V(40-60 G y) \times t)
\end{gathered}
$$

onde: bGH é o nível basal de GH; V(0-20 Gy), V(20-40 Gy) e V(40-60 Gy) são os volumes porcentuais do hipotálamo irradiado de 0-20 Gy, 20-40 Gy e 40-60 Gy, respectivamente; $\mathrm{t}$ é o tempo após a irra$\operatorname{diação}^{(19)}$.

Adan et al. também demonstraram, em crianças, que as concentrações de IGF-I após irradiação é dose dependente. As menores concentrações foram encontradas em pacientes que receberam as maiores doses. No entanto, essa correlação não foi encontrada com IGFBP-3, não havendo ainda uma justificativa para tal ${ }^{(20)}$.

Clayton e Shalet avaliaram dose e tempo de forma conjunta. Os pacientes receberam doses que variaram de 27 a 47,5 Gy (16 frações durante três semanas) e foram testados apenas para GH até quase 20 anos após a radioterapia. Um terço dos pacientes teve resultados normais dentro dos primeiros cinco anos em comparação com $16 \%$ após cinco anos. Análises múltiplas de regressão linear mostraram que dose e tempo a partir da irradiação tiveram influência significativa nos picos de GH. A incidência tardia de deficiência de $\mathrm{GH}$ foi semelhante em pessoas submetidas a doses menores ou maiores de irradiação (após cinco anos), mas a velocidade de aparecimento de deficiência nos primeiros anos foi dependente da dose, ou seja, quanto maior esta, mais rapidamente ocorria deficiência ${ }^{(\mathbf{2 1})}$.

Brauner e Rappaport constataram que quanto mais jovem o paciente e quanto menor o período de administração de irradiação, maior o risco de deficiência de $\mathrm{GH}^{(\mathbf{1 7})}$.

Um grupo de pacientes foi acompanhado após a irradiação e separado em três grupos: o primeiro grupo foi tratado com radioterapia convencional para algum tipo de doença hipofisária, com dose que variou de 20 Gy em oito frações por 11 dias até 45 Gy em 15 frações por 21 dias. O segundo grupo recebeu irradiação corporal total para malignidades hematológicas com 12 Gy em seis frações por três dias, e o terceiro grupo foi irradiado para algum tipo de tumor cerebral com 30 Gy em oito frações por 11 dias. Em relação à deficiência de TSH no primeiro grupo, foram notadas discrepâncias de acordo com as diferentes doses (Gráfico 1).

Além do TSH, foram avaliadas ACTH e FSH/LH, com resultados semelhantes. Já a deficiência de GH foi universal, por volta de cinco anos com dose de 35-45 Gy.
Resultados semelhantes foram observados nos grupos que receberam 30 Gy em oito frações por 11 dias e 45 Gy em 15 frações por 21 dias. Por outro lado, no grupo que recebeu 12 Gy em seis frações por três dias não houve disfunção hipofisária. Portanto, provavelmente não só a dose influencia, mas também o esquema de fracionamento da dose $\mathrm{e}^{(22)}$. Outros estudos mencionam que, com fração de irradiação maior num período mais curto de tempo, aumentará o impacto radiobiológico no eixo hipotálamo-hipofisário $^{(3,4)}$.

Pacientes que recebem irradiação em diferentes partes do corpo devem ter a dose efetiva biológica calculada, a fim de se ter uma estimativa de quanto o eixo hipotálamo-hipofisário recebeu de fato, assim permitindo uma comparação uniforme entre os pacientes. A fórmula utilizada é:

$$
B E D=D \times\{1+d /(\alpha / \beta)\}
$$

onde: D é a dose total; d é o tamanho da fração; $\alpha / \beta$ reflete a resposta do tecido à radiação.

As BED parciais também poderão ser calculadas separadamente quando a radioterapia envolve esquemas ou frações diferentes. As BED parciais podem ser somadas para se obter uma BED total ${ }^{(\mathbf{2 , 2 3})}$.

\section{CONSIDERAÇÕES FINAIS}

Alguns serviços apresentam estratégias para prevenir o hipopituitarismo decorrente de radioterapia para o eixo adeno-hipofisário. Uma delas é a tentativa em reduzir o tamanho tumoral com medicamentos,

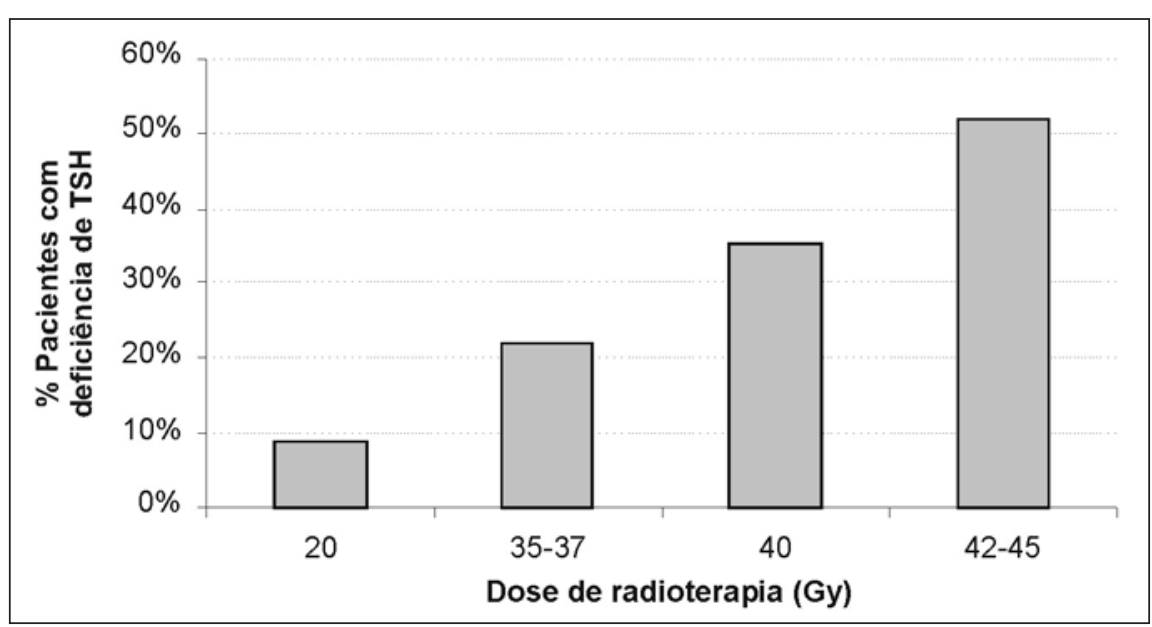

Gráfico 1. Deficiências de TSH dose dependente(22). 
antes de submeter o paciente a cirurgia e possível radioterapia. Outra forma é por meio de técnicas cirúrgicas, que têm como objetivo a adenomectomia seletiva ${ }^{(\mathbf{1})}$.

Apesar de a irradiação ser bem reconhecida como causa de hipopituitarismo, pacientes tratados em diversos setores nem sempre recebem acompanhamento do setor de endocrinologia. É essencial que esse grupo de pacientes seja tratado de forma conjunta, tanto com endocrinologistas como com oncologistas, otimizando a terapia e posterior qualidade de vida, com as devidas reposições hormonais.

\section{REFERÊNCIAS}

1. Lamberts SWJ, de Herder WW, van der Lely AJ. Pituitary insufficiency. Lancet 1998;352:127-34.

2. Darzy KH, Aimaretti G, Wieringa G, Gattamaneni HR, Ghigo E, Shalet SM. The usefulness of the combined growth hormone (GH)-releasing hormone and arginine stimulation test in the diagnosis of radiation-induced GH deficiency is dependent on the post-irradiation time interval. J Clin Endocrinol Metab 2003;88:95-102.

3. Toogood AA, Ryder WDJ, Beardwell CG, Shalet SM. The evolution of radiation-induced growth hormone deficiency in adults is determined by the baseline growth hormone status. Clin Endocrinol 1995;43:97-103.

4. Toogood AA, Nass RM, Pezzoli SS, O’Neill PA, Thorner MO, Shalet SM. Preservation of growth hormone pulsatility despite pituitary pathology, surgery, and irradiation. J Clin Endocrinol Metab 1997;82:2215-21.
5. Lissett CA, Saleem S, Rahim A, Brennan BMD, Shalet SM. The impact of irradiation on growth hormone responsiveness to provocative agents is stimulus dependent: results in 161 individuals with radiation damage to the somatotropic axis. J Clin Endocrinol Metab 2001;86:663-8.

6. Boguszewski CL. Análise crítica dos testes para o diagnóstico da deficiência do hormônio do crescimento. In: Vilar L. Endocrinologia clínica. 2a ed. Rio de Janeiro, RJ: Medsi, 2001:137-42.

7. Nivot S, Benelli C, Clot JP, et al. Nonparallel changes of growth hormone $(\mathrm{GH})$ and insulin-like growth factor-I, insulin-like growth factor binding protein-3, and $\mathrm{GH}$ binding protein, after craniospinal irradiation and chemotherapy. J Clin Endocrinol Metab 1994;78:597-601.

8. Lam KS, Tse VK, Wang C, Yeung RT, Ma JT, Ho $\mathrm{JH}$. Early effects of cranial irradiation on hypothalamic-pituitary function. J Clin Endocrinol Metab 1987;64:418-24.

9. Constine LS, Woolf PD, Cann D, et al. Hypothalamic-pituitary dysfunction after radiation for brain tumors. N Engl J Med 1993;328:87-94.

10. Trainer PJ, Besser GM. The Bart's endocrine protocols. London: Churchill Livingstone, 1995:3-18.

11. Littley MD, Shalet SM, Beardwell CG. Radiation and hypothalamic-pituitary function. Baillieres Clin Endocrinol Metab 1990;4:147-75.

12. Frajese G, Drake WM, Loureiro RA, et al. Hypothalamo-pituitary surveillance imaging in hypopituitary patients receiving long-term growth hormone replacement therapy. J Clin Endocrinol Metab 2001;86:5172-5.

13. Stafford SL, Pollock BE, Leavitt JA, et al. A study on the radiation tolerance of the optic nerves and chiasm after stereotatic radiosurgery. Int J Radiat Oncol Biol Phys 2003;55:1177-81.

14. Benbassat CA, Olchovsky D. Prolactinoma and other head and neck tumors after scalp irradiation. South Med J 2003;96:209-11.
15. Brennan BM, Rahim A, Blum WF, Adams JA, Eden OB, Shalet SM. Hyperletinaemia in young adults following cranial irradiation in childhood: growth hormone deficiency or leptin insensitivity? Clin Endocrinol 1999;50:163-9.

16. Nishioka H, Hirano A, Haraoka J, Nakajima N. Histological changes in the pituitary gland and adenomas following radiotherapy. Neuropathology 2002;22:19-25.

17. Brauner R, Rappaport R. Endocrine complications caused by cranial irradiation. Ann Pediatr 1989;36: $419-24$.

18. Pai HH, Thornton A, Katznelson L, et al. Hypothalamic/pituitary function following high dose conformal radiotherapy to the base of skull: demonstration of a dose effect relationship using dose-volume histogram analysis. Int J Radiat Oncol Biol Phys 2001;49:1079-92.

19. Merchant TE, Goloubeva O, Pritchard DL, et al. Radiation dose-volume effects on growth hormone secretion. Int J Radiat Oncol Biol Phys 2002;52: 1264-70.

20. Adan L, Trivin C, Sainte-Rose C, Zucker JM, Hartmann O, Brauner R. GH deficiency caused by cranial irradiation during childhood: factors and markers in young adults. J Clin Endocrinol Metab 2001; 86:5245-51.

21. Clayton PE, Shalet SM. Dose dependency of time of onset of radiation-induced growth hormone deficiency. J Pediatr 1991;118:226-8.

22. Littley MD, Shalet SM, Beardwell CG, Robinson EL, Sutton ML. Radiation-induced hypopituitarism is dose-dependent. Clin Endocrinol 1989;31:36373.

23. Schmiegelow M, Lassen S, Poulsen HS, et al. Cranial radiotherapy of childhood brain tumours: growth hormone deficiency and its relation to the biological effective dose of irradiation in a large population based study. Clin Endocrinol 2000;53: $191-7$. 